\title{
Interferon- $\gamma$ release assays do not identify more children with active tuberculosis than the tuberculin skin test
}

\author{
B. Kampmann, E. Whittaker, A. Williams, S. Walters, A. Gordon, N. Martinez-Alier, \\ B. Williams, A.M. Crook, A-M. Hutton and S.T. Anderson
}

ABSTRACT: Data are lacking on the performance of interferon- $\gamma$ release assays (IGRAs) in children. Although IGRAs are recommended for screening for latent tuberculosis infection (LTBI), many clinicians wish to employ them as a diagnostic test for active tuberculosis (TB). The objective of the present study was to compare the performance of the two commercially available IGRAs and the tuberculin skin test (TST) side-by-side in children with active TB and LTBI.

In a prospective study, 209 children were investigated for active $(n=91)$ or latent TB $(n=118)$. TST, QuantiFERON-TB Gold In-tube (QFG-IT; Cellestis, Carnegie, Australia) and T-SPOT.TB (Oxford Immunotec, Abingdon, UK) assays were simultaneously used.

For culture-confirmed active TB, the sensitivity of the TST was $83 \%$, compared with $80 \%$ for QFG-IT and $58 \%$ for T-SPOT.TB. IGRAs did not perform significantly better than TST, although QFG-IT was significantly better than T-SPOT.TB. The agreement between QFG-IT and T-SPOT.TB in culture-confirmed TB was poor at $66.7 \%$. In LTBI, the agreement between QFG-IT and TSPOT.TB was very good (92\%) with moderate agreement between TST and T-SPOT.TB (75\%) and QFG-IT and TST (77\%).

A negative interferon- $\gamma$ release assay should not dissuade paediatricians from diagnosing and treating presumed active tuberculosis. If used for diagnosis of latent tuberculosis infection, interferon- $\gamma$ release assays could significantly reduce the numbers of children receiving chemoprophylaxis. Very good concordance between both tests was found.

\section{KEYWORDS: Children, interferon- $\gamma$ release assays, skin test, tuberculosis}

$\mathbf{T}$ he identification of Mycobacterium tuberculosis-specific antigens has led to the successful development of commercially available immunoassays that are able to distinguish the production of interferon (IFN)- $\gamma$ in response to $M$. tuberculosis infection from the bacillus Calmette-Guérin (BCG) vaccine effect, unlike the tuberculin skin test (TST) [1-3]. For the purposes of screening for latent tuberculosis infection (LTBI), and to avoid unnecessary chemoprophylaxis, these blood-based IFN- $\gamma$ release assays (IGRAs) are now widely recommended for use in adults and children with a positive TST, and have found their way into national guidelines in several countries, including the UK [4]. In the UK, however, no recommendations have been made regarding which of the two commercially available assays to use and the guidelines do not suggest using IGRAs for the diagnosis of active tuberculosis (TB).

However, clinicians (and paediatricians in particular) are searching for improved diagnostic tools [1] and have been exploring IGRAs for immunodiagnosis of active TB, since microbiological confirmation is the exception rather than the rule in children due to the paucibacillary nature of their disease [2].

Of the large number of publications assessing the performance of IGRAs versus the TST, most relate to adult cohorts, and the majority have only evaluated one of the two commercially available IGRAs in comparison with the TST. The few studies comparing both IGRAs with the TST have small paediatric cohorts including a minority of children with active TB $[3,5,6]$. Most investigators have chosen to analyse the combined results

\section{AFFILIATIONS}

For affiliations, please see the Acknowledgements section.

\section{CORRESPONDENCE}

B. Kampmann, Academic Dept of Paediatrics, 2nd Floor WrightFleming Building, Imperial College London, St. Mary's Campus, Norfolk Place, London W2 1PG, UK. Fax: 442075943894

E-mail: b.kampmann@imperial.ac.uk

Received:

October 102008

Accepted after revision: January 122009

\section{SUPPORT STATEMENT}

This work was supported by consumable grants from the PEEL Foundation (London, UK) and the European Society for Paediatric Infectious Diseases (Geneva, Switzerland). B. Kampmann is funded by a Career Development Fellowship (GR 077273) from the Wellcome

Trust (London), is supported by the Biomedical Research Centre at Imperial College London and is a coinvestigator at the Centre for Respiratory Infection at Imperial College London (London, UK). S.T. Anderson is supported by a European Union grant: Aid for poverty-related diseases in developing countries (EuropeAid/121404/C/G/Multi). The funding sources had no role in study design, data collection, data analysis, data interpretation or writing of the report.

STATEMENT OF INTEREST A statement of interest for S.T. Anderson can be found at www.erj.ersjournals.com/misc/ statements.dtl 
of performance of IGRAs versus TST in active TB as well as LTBI. The aim of these studies has been to assess concordance of IGRAs with TST, and the published data have generally shown superior sensitivity of IGRAs in LTBI [3, 7, 8]. Some studies have also demonstrated higher diagnostic sensitivity and specificity of IGRAs over TST in active TB [9-11]. However, several recent reviews have concluded that performance of IGRAs in young children and immunocompromised individuals deserve further investigation and that a gap of knowledge persists in this area [12, 13].

It was postulated that the performance of IGRAs in children might be discrepant between active TB and LTBI, since the underlying immunological phenomena are probably different.

The aim of the present study was to describe the performance of both commercially available IGRAs that are recommended by the National Institute of Health and Clinical Excellence (NICE) in the UK, side-by-side with the TST, in the setting of active and latent TB. Children were recruited from two National Health Service (NHS) hospital trusts in north-west London, UK, where the TB incidence of the local population varies $40-100$ per 100,000 population [14].

\section{METHODS}

\section{Study participants}

Study approval was obtained from the ethics committees of Imperial College Healthcare NHS Trusts and the North West London Hospitals Trust. Written consent was provided by all study participants or their parents or legal guardians. Verbal consent was also sought from children aged $>10$ yrs.

Between February 2006 and February 2008, children with suspected active TB or LTBI attending the inpatient and outpatient services at Imperial College Healthcare NHS Trust St Mary's Hospital or North West London Hospitals NHS Trust were prospectively enrolled.

\section{Inclusion criteria}

All children aged between 2 months and 16 yrs who were investigated for active TB were included, as were referrals for contact tracing after exposure to a case of active TB.

\section{Definition of study groups}

Active TB

Cases of active TB were defined according to the following three categories. 1) Definite TB: all children in this group had culture-confirmed TB. 2) Probable TB: all children in this group had (in the absence of culture confirmation) symptoms and signs of active TB; abnormal radiography consistent with $\mathrm{TB}$ and/or abnormal cerebro-spinal fluid (CSF) consistent with tuberculous meningitis; response to TB therapy; and either a history of TB contact or a history of travel to TB-endemic countries within the previous 24 months. 3) Possible TB: signs and symptoms consistent with active $\mathrm{TB}$ and existing risk factors for active TB, such as history of TB contact or history of travel to TB-endemic countries. Subsequently, however, the treating paediatrician made an alternative diagnosis or felt that TB was unlikely. Therefore, none of the children in this group ever received TB treatment and none subsequently developed active TB.

\section{$\angle T B /$}

Children with suspected LTBI were children with documented exposure to active TB. Results for these children were stratified according to their BCG vaccination record by a TST $\geqslant 15 \mathrm{~mm}$ (BCG vaccinated) or a TST of $\geqslant 6 \mathrm{~mm}$ (not BCG vaccinated), following the UK guidelines.

Consent for the study, and demographic and clinical data were obtained by the paediatricians in charge of the patients' clinical care. All blood samples were handled by laboratory staff blinded to the clinical status of the patients. Results of IGRA testing were not available to the treating physicians.

\section{Investigations for active TB}

Children admitted with suspected active pulmonary TB (PTB) had sputum samples and/or gastric washings collected as per routine protocol. Any child with suspected TB meningitis had CSF sent for microscopy and culture. For other sites of presumed disseminated disease, tissue biopsies were obtained where possible and appropriate. Any extrapulmonary manifestation of TB is referred to as EPTB.

Tuberculin skin testing

TSTs were sited by the medical or TB nursing staff, according to routine hospital procedure.

Two units of purified protein derivative RT23 (Staten Serum Institute, Copenhagen, Denmark) were applied to the volar surface of the left lower forearm according to the intradermal Mantoux method. The transverse diameter of skin induration was recorded in millimetres $48-72 \mathrm{~h}$ later. In line with the cutoffs selected by NICE for interpretations of TST results for LTBI, TST results were stratified into two groups of $\geqslant 6 \mathrm{~mm}$, and $\geqslant 15 \mathrm{~mm}$, depending on the absence or presence of previous BCG vaccination. For active TB, sensitivities of cutoffs of $15 \mathrm{~mm}$ and $10 \mathrm{~mm}$ were evaluated.

\section{Laboratory assays}

Peripheral blood samples were obtained simultaneously for both assays by paediatric phlebotomists who were trained in the use of the specific blood collection tubes, according to the manufacturer's recommendations. All blood samples were processed within $4 \mathrm{~h}$ of phlebotomy, as recommended.

The commercially available T-SPOT.TB (Oxford Immunotec, Abingdon, UK) and the QuantiFERON-TB Gold In-tube (QFGIT) system (Cellestis, Carnegie, Australia) were used in all assays.

\section{T-SPOT.TB}

Blood samples were collected in 4-mL CPT tubes (BD Bioscience, Oxford, UK) and peripheral blood mononuclear cells (PBMCs) were prepared within $2 \mathrm{~h}$, following standard procedures. Commercially available T-SPOT.TB kits were used for all assays. All procedures, including interpretation of $\mathrm{T}$ SPOT.TB results, were carried out according to the manufacturer's recommendations. Absolute numbers of spot-forming cells (SFCs) were counted using an Elispot counter (AID, Strassberg, Germany) and numbers of antigen-specific SFCs in positive T-SPOT.TB assays were plotted following subtraction of numbers in the negative control well. 


\section{QFG-IT}

The commercially available blood bottles and ELISA kits were used for all assays, following manufacturer's recommendations. IFN- $\gamma$ values for TB-specific antigens, mitogen and negative control were calculated by the Cellestis software program. The cut-off value for a positive result was set at $>0.35$ IU of IFN- $\gamma$, as recommended by the manufacturers. The test result was considered to be indeterminate for TB antigen responsiveness if the IFN- $\gamma$ level was $<0.35 \mathrm{IU} \cdot \mathrm{mL}^{-1}$ in the TB antigen well and the mitogen minus nil was $<0.5 \mathrm{IU} \cdot \mathrm{mL}^{-1}$ or if the nil value was $>8.0 \mathrm{IU} \cdot \mathrm{mL}^{-1}$. Absolute levels of antigen specific IFN- $\gamma$ were calculated by subtracting the value of the negative control from the antigen-specific value.

\section{Statistical analysis}

Sensitivity of the assays was measured by the proportion of positive results identified in the whole cohort and the

\section{TABLE 1 Demographic details of the study cohort}

\begin{tabular}{lcc} 
& Active TB & LTBI \\
\hline Subjects & 91 & 118 \\
Age yrs & & \\
$<1$ & $2(2.2)$ & $18(16.3)$ \\
$1-2$ & $7(7.7)$ & $8(6.7)$ \\
$2.1-5$ & $18(19.8)$ & $31(26.0)$ \\
$5.1-10$ & $18(19.8)$ & $22(18.0)$ \\
10.1-16 & $46(50.5)$ & $39(33.0)$ \\
Sex & & \\
Female & $47(51.6)$ & $57(48.3)$ \\
Male & $44(48.4)$ & $61(51.7)$ \\
Ethnicity & & \\
Black African & $49(54.4)$ & $50(42.0)$ \\
South Asian & $21(23.3)$ & $39(33.0)$ \\
South-east Asian & $6(6.6)$ & $9(8.0)$ \\
Afro-Caribbean & $0(0)$ & $4(3.0)$ \\
Arab & $7(7.7)$ & $3(2.5)$ \\
Mixed & $1(0.01)$ & $7(6.0)$ \\
Caucasian & $7(7.7)$ & $6(5.0)$ \\
Country of birth & & \\
UK & $30(33.0)$ & $50(42.3)$ \\
Other & $61(67.0)$ & $68(57.7)$ \\
Known TB contact & & \\
Yes & & \\
No & & \\
Unknown & $40(44.0)$ & $102(86.0)$ \\
BCG vaccinated & $47(51.6)$ & $13(11.0)$ \\
Yes & $4(4.4)$ & $3(3.0)$ \\
No & & \\
Unknown & $55(60.4)$ & $87(74.0)$ \\
Yes & $23(25.3)$ & $17(14.0)$ \\
No & $13(14.3)$ & $14(12.0)$ \\
Unknown & & \\
\hline & $58(63.7)$ & $55(47.0)$ \\
\hline & $25(27.5)$ & $53(44.5)$ \\
\hline & & $10(8.5)$ \\
\hline
\end{tabular}

Data are presented as $n$ or $n(\%)$. TB: tuberculosis; LTBI: latent TB infection. BCG: bacillus Calmette-Guérin. following subgroups: active TB (which was further categorised as "definite", "probable", "definite or probable" and "possible $^{\prime \prime}$ ) and LTBI, stratified by BCG vaccination status.

Failed or indeterminate assay results were excluded from statistical analysis for sensitivity or agreement between tests.

Agreement between assays was measured by total per cent agreement and the $\kappa$ statistic. The strength of agreement was defined as poor with $\kappa \leqslant 0.2$, fair if $\kappa$ was $0.21-0.4$, moderate if $\kappa$ was $0.41-0.6$, good if $\kappa$ was $0.61-0.8$ and very good if $\kappa$ was $0.8-1$ [15].

\section{RESULTS}

Between February 2006 and February 2008, 209 children who attended the NHS paediatric services for active or latent TB were enrolled into the study.

\section{Demography}

The mean (range) age of children presenting with active TB was $9.2 \mathrm{yrs}$ (6 months to $15 \mathrm{yrs}$ ), and that of children investigated for LTBI was $6.9 \mathrm{yrs}$ (3 months to $16 \mathrm{yrs}$ ). Table 1 summarises the demographic details of the cohort.

The male/female ratio was 1:1 for the active cases and 1:0.9 for the LTBI cohort. In line with the mixed community living in north-west London and attending NHS facilities, most children were from ethnic minorities. The majority of children (129 out of $209 ; 62 \%$ ) were born outside of the UK.

In total, $68 \%$ (142 out of 209) of children had a known household contact, with 118 (56.45\%) investigated for LTBI and $91(43.55 \%)$ for active TB.

The majority of children were BCG vaccinated (142 (68\%) out of 209). Children were not routinely tested for HIV, but all children with active TB and additional epidemiological risk factors for the presence of HIV $(n=35)$ were screened for HIV and all found to be negative.

\section{Test results for all children}

Of the 209 children enrolled, 204 had available results for TST, 206 had a T-SPOT.TB sample analysed and 209 had available data for QFG-IT. The results per test conducted are summarised in table 2, stratified for children above and below 5 yrs of age.

\section{Failures of QFG-IT and T-SPOT.TB}

There were no technical failures with the QFG-IT, but 14 (6.7\%) indeterminate results were obtained; eight in active TB and six in LTBI. The mean (range) age of children with indeterminate results was $6.4(0.5-16)$ yrs. There were 18 failures $(8.7 \%)$ of the T-SPOT.TB assay, consisting of sample errors prior to generating the PBMCs, such as clot in the blood sample $(n=4)$, insufficient blood taken $(n=3)$, and uninterpretable results $(n=11)$ after processing of PBMCs because of high backgrounds $(n=9)$ or low phytohaemagglutinin response $(n=2)$. The mean age of children whose sample failed the T-Spot.TB was $4.74(0.7-16)$ yrs. The mean age of the children with failed tests was not significantly different from the mean age of the 
TABLE 2 Performance of interferon- $\gamma$ release assays and tuberculin skin test (TST) in all children according to age

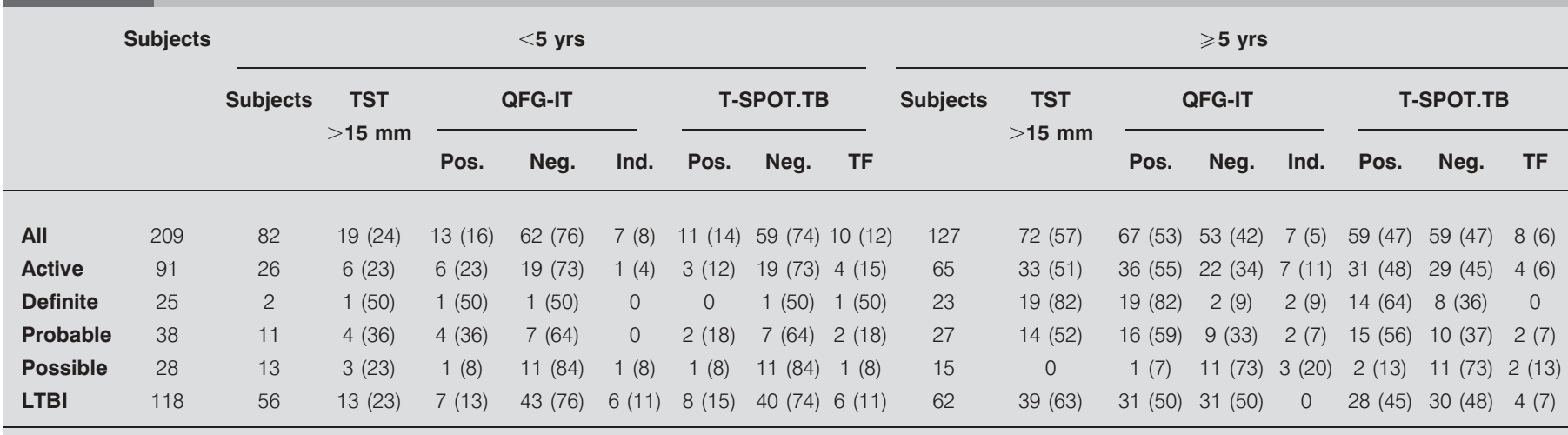

Data are presented as n or n (\%). QFG-IT: QuantiFERON-TB Gold In-tube system (Cellestis, Carnegie, Australia); pos.: positive; neg.: negative; ind.: indeterminate result; TF: test failure; LTBI: latent tuberculosis infection. T-SPOT.TB is manufactured by Oxford Immunotec, Abingdon, UK.

children with successful tests. Failed or indeterminate test results were not included in the further analysis.

\section{Performance of tests in children with active TB}

The cohort of children that was investigated for active TB $(n=91)$ was divided into three subgroups, as specified in the Methods section. All children classified as definite (culture proven) TB $(n=25)$ and probable TB $(n=38)$ received treatment for active TB. No children classified as possible TB $(n=28)$ at study entry subsequently received anti-TB therapy. Additionally, results are shown for the combination of definite and probable cohorts in order to illustrate the potential value of IGRAs and TST in the reality of paediatric practice, where TB treatment might be administered on clinical grounds in the absence of culture confirmation (table 3 ).

Sensitivities for the TST were calculated using two different cut-offs of $15 \mathrm{~mm}$ and $10 \mathrm{~mm}$ to examine the relative effect on sensitivity. In active, culture-confirmed TB the sensitivity was $83 \%$ for a TST $\geqslant 15 \mathrm{~mm}, 87.5 \%$ for a TST $\geqslant 10 \mathrm{~mm}, 80 \%$ for
QFG-IT and 58\% for T-SPOT.TB. Since the different cut-offs for TST did not result in a significant difference, further analysis was pursued with the cut-off of $15 \mathrm{~mm}$, which is likely to be more specific for $M$. tuberculosis infection than a cut-off of $10 \mathrm{~mm}$.

IGRAs did not perform significantly better than the TST in this group, but QFG-IT performed significantly better than TSPOT.TB $(p=0.015)$ in identifying culture-confirmed, active TB. There was no significant difference between any test in predicting probable $(p=0.62)$ or possible TB $(p>0.99)$. When results for definite and probable active TB were combined, TST $\geqslant 15 \mathrm{~mm}$ had a sensitivity of $60 \%$, QFG-IT of $64 \%$ and TSPOT.TB of $50 \%$, as summarised in figure 1 a.

In order to test for improved sensitivity by combining tests, the sensitivity for TST $\geqslant 15 \mathrm{~mm}$ combined with QFG-IT or TSPOT.TB was calculated. In definite TB, a combination of TST and QFG-IT $(\mathrm{n}=22)$ achieved 91\% sensitivity, compared with $100 \%$ for TST plus T-SPOT.TB $(n=22)$. For probable TB,

TABLE 3 Summary of interferon- $\gamma$ release assay and tuberculin skin test (TST) results in children with active tuberculosis (TB) and latent tuberculosis infection (LTBI)

\begin{tabular}{|c|c|c|c|c|c|c|c|c|c|}
\hline & \multicolumn{3}{|c|}{ TST $\mathrm{mm}$} & \multicolumn{3}{|c|}{ QFG-IT } & \multicolumn{3}{|c|}{ T-SPOT.TB } \\
\hline & $\geqslant 15$ & $\geqslant 10$ & $<6$ & Pos. & Neg. & Ind. & Pos. & Neg. & TF \\
\hline Definite & 20/24 (83.3) & $21 / 24(87.5)$ & 2/24 (8.3) & 20/25 (80.0) & $3 / 25$ (12.0) & 2/25 (8.0) & $14 / 24$ & 9/24 (37.5) & $1 / 24(4.2)$ \\
\hline Probable & $17 / 38(44.7)$ & 24/38 (63.1) & 9/38 (23.6) & 20/38 (52.6) & $16 / 38$ & 2/38 (5.2) & $17 / 38(44.7)$ & $17 / 38(44.7)$ & 4/38 (10.5) \\
\hline \multicolumn{10}{|l|}{ LTBI } \\
\hline BCG vaccinated & $38 / 84(45.2)$ & $51 / 84(60.7)$ & $59 / 84(70.2)$ & 28/87 (32.1) & $53 / 87(60.9)$ & $6 / 87(6.9)$ & $25 / 85(29.4)$ & $51 / 85(60.0)$ & 9/85 (10.6) \\
\hline Not BCG vaccinated & $14 / 30(46.7)$ & $17 / 30(56.7)$ & $19 / 30(63.3)$ & $10 / 31(32.2)$ & $21 / 31(67.8)$ & $0 / 31(0.0)$ & $11 / 31$ & $19 / 31$ & $1 / 31$ \\
\hline
\end{tabular}

Data are presented as n (\%).QFG-IT: QuantiFERON-TB Gold In-tube system (Cellestis, Carnegie, Australia); pos.: positive; neg.: negative; ind.: indeterminate result; TF: test failure; BCG: bacillus Calmette-Guérin. T-SPOT.TB is manufactured by Oxford Immunotec, Abingdon, UK. 

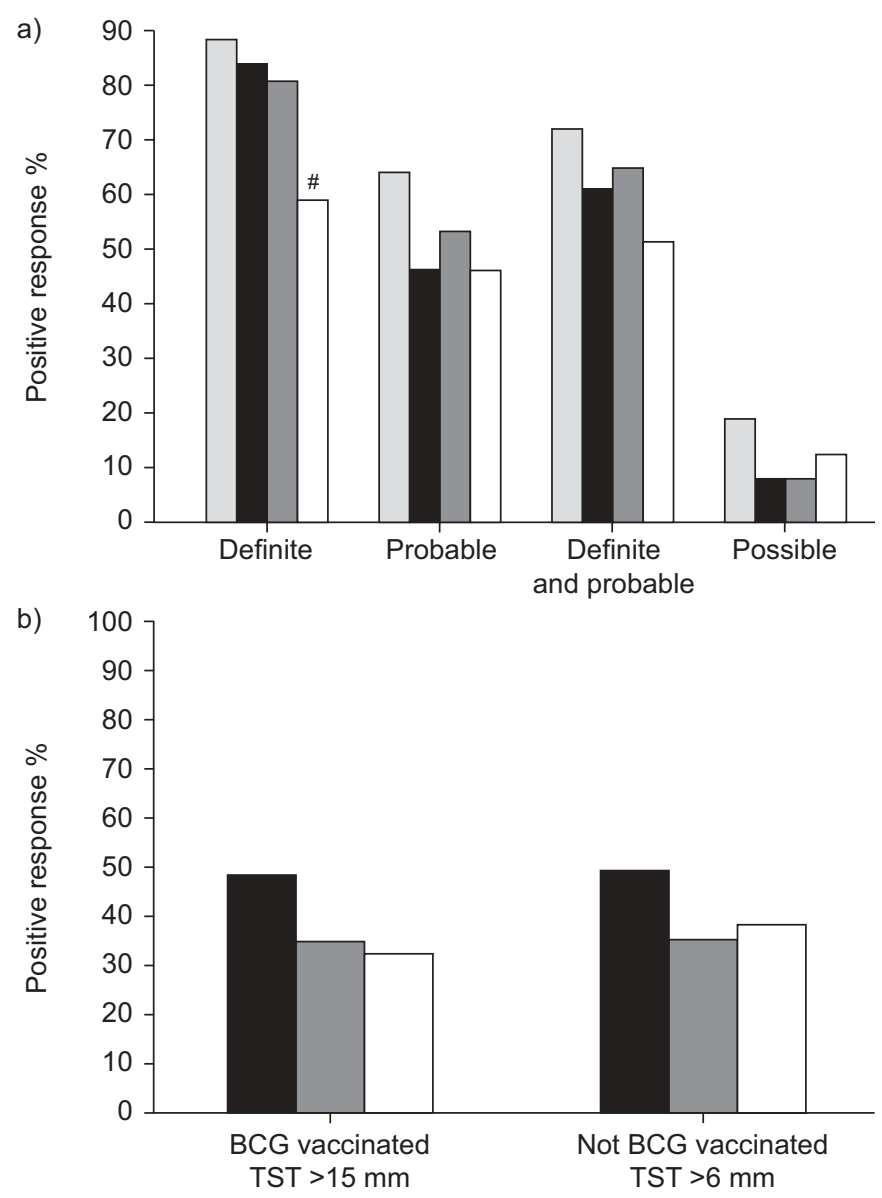

FIGURE 1. a) Percentage of positive responses in tuberculin skin test (TST) and interferon- $\gamma$ release assays (IGRAs) according to TST cut-off and categories of active tuberculosis (TB). $:$ : TST $\geqslant 10 \mathrm{~mm}$; $\mathbf{\square}:$ TST: $\geqslant 15 \mathrm{~mm}$; $\mathbf{\square}:$ QFG-IT; $\square:$ TSPOT.TB. ${ }^{*}: p=0.012$ sensitivity of QuantiFERON-TB Gold In-tube (QFG-IT) system (Cellestis, Carnegie, Australia) and T-SPOT.TB (Oxford Immunotec, Abingdon, UK; $80 \%$ versus $58 \%$ ) in culture-confirmed TB. b) Percentage of positive responses in TST and IGRAs in latent TB infection (LTBI) according to bacillus Calmette-Guérin (BCG) status. BCG vaccinated: $n=87$, TST cut-off $\geqslant 15 \mathrm{~mm}$; not $B C G$ vaccinated: $\mathrm{n}=31$, TST cut-off $>6 \mathrm{~mm}$, in accordance with National Institute of Health and Clinical Excellence guidelines [4]. ㅍ: : TST; 1 : QFG-IT; $\square$ : T-SPOT.TB.

sensitivities were $64 \%(n=36)$ versus $59 \% \quad(n=34)$ and for combined definite and probable $\mathrm{TB}$, sensitivities were $74 \%$ $(n=58)$ and $75 \%(n=56)$ respectively.

Of the 25 children with culture-confirmed TB, 15 presented with PTB and 10 with EPTB. A TST $\geqslant 15 \mathrm{~mm}$ was present in $93 \%$ of children with PTB, but in only $60 \%$ in children with EPTB. The sensitivity of QFG-IT was unaffected by the manifestation of $\mathrm{TB}(80 \%$ in both $\mathrm{PTB}$ and EPTB $)$, but sensitivity of the T-SPOT.TB in EPTB was lower than for PTB (40\% versus $67 \%$ ).

At the time of diagnosis, a TST of $<6 \mathrm{~mm}$ was found in two $(8 \%)$ out of 24 , a negative QFG-IT in three $(12 \%)$ out of 25 and a negative T-SPOT.TB in nine (39\%) out of 23 of children with culture-confirmed TB.

Of those children with possible $\mathrm{TB}, 79 \%$ had a negative result in all three tests.

\section{Results for children with LTBI}

Of 118 children investigated for LTBI, the majority were BCG vaccinated $(87(74 \%)$ out of 118$)$, as summarised in table 3 . More children had a positive TST than IGRAs, but a record of BCG vaccination was not associated with a positive result in either the TST or IGRAs (TST $\geqslant 15 \mathrm{~mm}$ in $45.2 \%$ of BCGvaccinated children versus TST $\geqslant 6 \mathrm{~mm}$ in $46.7 \%$ in BCGunvaccinated children; positive QFG-IT of $32 \%$ in BCGvaccinated children versus $32.2 \%$ in BCG-unvaccinated children; positive T-SPOT.TB in $29.4 \%$ of BCG-vaccinated children versus $35.5 \%$ in BCG-unvaccinated children; nonsignificant).

\section{Concordance between all three tests}

To measure the agreement between all tests in both active and latent TB, $\kappa$ statistics were calculated for TST $\geqslant 15 \mathrm{~mm}$ versus both IGRAs as well as agreement between the two IGRAs.

The concordance between the two IGRAs was higher $(88 \%, \kappa$ $0.75)$ than between IGRAs and TST (78.5\% for QFG-IT versus TST, $\kappa 0.57$; and $73.5 \%$ for T-SPOT.TB versus TST, $\kappa 0.46$ ), as shown in table 4 .

In active TB, overall agreement between IGRAs was good $(82.7 \%, \kappa 0.66)$. However, the agreement between QFG-IT and T-SPOT.TB in children with culture-confirmed TB was poor $(66.7 \%, \kappa 0.15)$. QFG-IT correctly identified a significantly larger number of children with definite TB $(\mathrm{p}=0.012)$ compared with T-SPOT.TB.

In LTBI, very good agreement was found between both IGRAs $(92 \%, \kappa 0.82)$, but the agreement between TST and IGRAs was only moderate (TST versus T-SPOT.TB, к 0.49; TST versus QFGIT, к 0.53).

Presence or absence of BCG vaccination did not significantly influence the proportion of positive tests (fig. 1b) or the agreement between the IGRAs $(90 \%$ in BCG-vaccinated children versus $96 \%$ in BCG-unvaccinated children, nonsignificant), as shown in table 4 .

\section{Absolute values of TST and IGRAs}

In order to assess whether absolute values of TST induration and/or production of IFN- $\gamma$ in either IGRA could be used to distinguish between active and latent $\mathrm{TB}$, all results were analysed according to their actual numeric values and not just according to positive or negative results. A significant difference in millimetres of induration was seen between both definite (median (range) $20(6-35) \mathrm{mm}$ ) and probable TB (15 $(6-14) \mathrm{mm} ; \mathrm{p}=0.018)$ as well as between definite and latent TB (12.5 $(0-41) \mathrm{mm} ; \mathrm{p}=0.0003)$, as shown in figure $2 \mathrm{a}$.

As summarised in figure $2 b$ and $c$, there was no statistically significant difference between active and latent TB in absolute levels of secretion of IFN- $\gamma$ or in numbers of SFCs, independent of the antigen investigated.

\section{DISCUSSION}

The present report is the first paediatric study to evaluate, sideby-side, the two commercially available IGRAs with TST, analysing for both active and latent $\mathrm{TB}$ in a large paediatric cohort, since the introduction of the new TB guidelines in the UK. Very good agreement was found between the IGRAs in 
TABLE 4 Per cent concordance between tests and $\kappa$ coefficients

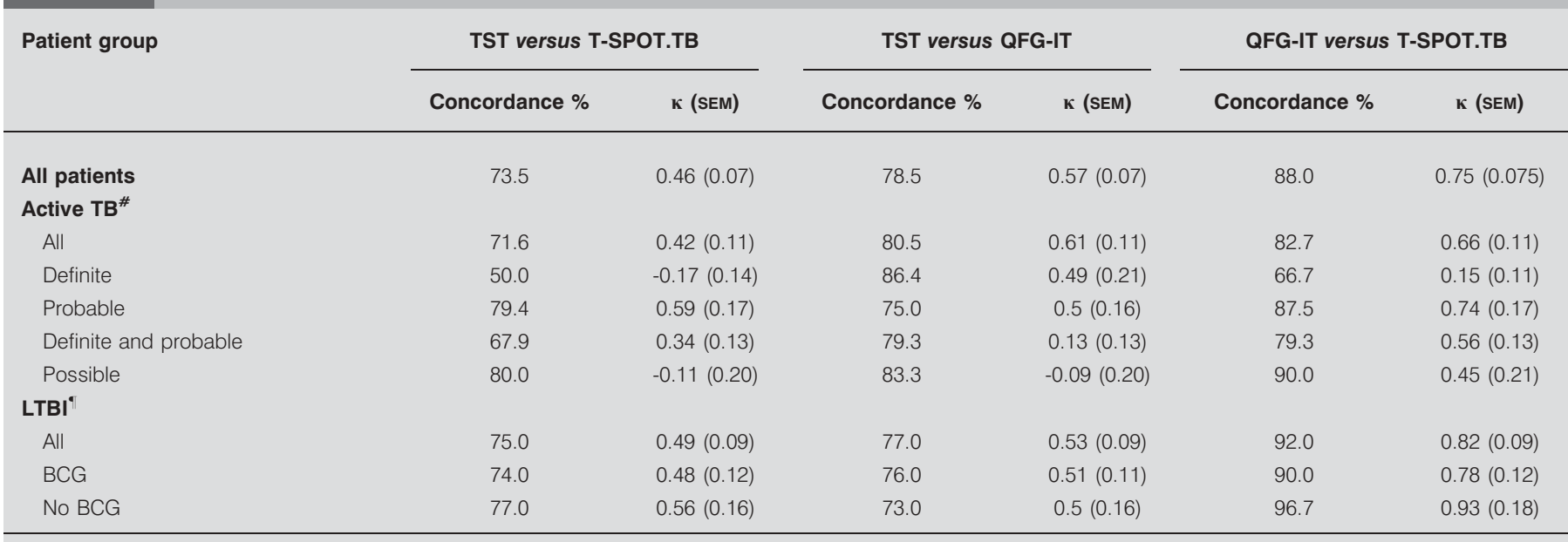

TST: tuberculin skin test; QFG-IT: QuantiFERON-TB Gold In-tube system (Cellestis, Carnegie, Australia); TB: tuberculosis; LTBI: latent TB infection; BCG: bacillus Calmette-Guérin. T-SPOT.TB is manufactured by Oxford Immunotec, Abingdon, UK. ${ }^{\#}: \mathrm{n}=91 ;{ }^{\bullet}: \mathrm{n}=118$.

the setting of LTBI, but low sensitivity of the T-SPOT.TB and, in particular, poor agreement between tests for the diagnosis of active TB were also found.

The diagnosis of active TB remains a challenge in paediatrics, as bacteriological confirmation is the exception rather than the rule, because of the paucibacillary nature of the disease and the relative difficulties in collecting adequate samples for microbiology [2]. Paediatricians around the globe will acknowledge that the majority of their patients are treated for TB in the absence of fulfilling the gold standard of bacteriological confirmation, and will instead receive a 6-month course of
TB treatment based on a combination of clinical signs and symptoms, suggestive radiology, history of household exposure or travel to TB-endemic countries, as well as the TST results [16]. Treatment is often justified, in retrospect, through the dramatic improvement of clinical symptoms.

In the present study, 91 children were enrolled and investigated for active TB, a third of which was culture confirmed. The calculated sensitivities for IGRAs and TST differ depending on whether only confirmed cases or children with probable TB were included, using a strict set of inclusion criteria. The current authors reasoned that it is important to assess a novel
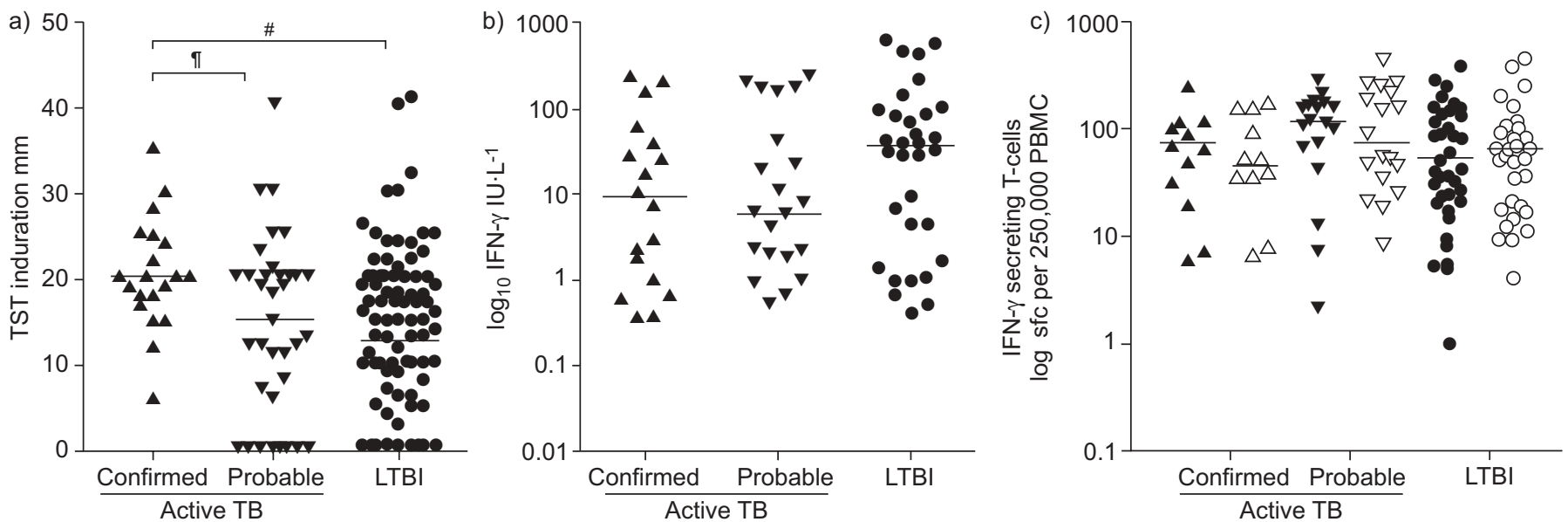

FIGURE 2. Absolute values of tuberculin skin test (TST) and interferon- $\gamma$ release assays (IGRAs) in active tuberculosis (TB) and latent TB infection (LTBI). a) TST induration in children with either active TB (culture confirmed $(n=20)$ or probable $(n=35))$ or LTBI $(n=118)$. Horizontal lines indicate median values. ${ }^{\#}: p=0.0003 ; ": p=0.0188$ b) Net concentration of interferon (IFN)- $\gamma$ (antigen-stimulated level minus control level of IFN- $\gamma$ ) from positive QuantiFERON-TB Gold In-tube (Cellestis, Carnegie, Australia) assays performed on patients with either active TB (culture confirmed $(n=18)$ or probable $(n=22))$ or LTBI $(n=36)$. Horizontal lines indicate median values. There were no statistically significant differences between the groups. c) Spot-forming cells (sfc) per 250,000 IFN- $\gamma$ producing T-cells in response to CFP-10 (closed symbols) and ESAT-6 (open symbols) antigens in T-SPOT.TB tests (Oxford Immunotec, Abingdon, UK) performed on patients with either active TB (culture confirmed ( $\mathrm{n}=13$ ) or probable disease $(n=18))$ or LTBI $(n=34)$. Results were converted into a $\log _{10}$ scale for ease of interpretation. There were no statistically significant differences between the groups. PBMC: peripheral blood mononuclear cell. 
diagnostic test by not only using the gold standard of cultureconfirmed TB, but also to assess its performance in the "reallife" situation of probable TB, since this evaluates the diagnostic potential of IGRAs more accurately in paediatric practice. As a result, a lower sensitivity of IGRAs in active TB may have been found compared with that reported from adult studies.

Young children are most vulnerable to developing active TB after a household contact, reflecting the immaturity of their immune responses. Primary TB elicits different immune responses from those seen in adults, in whom TB is usually caused by reactivation or reinfection [17]. As a consequence, TBspecific IFN- $\gamma$-producing T-cells might be present in lower numbers in children compared with adults [18-20], resulting in lower levels of IFN- $\gamma$, the target cytokine measured in IGRAs. As a consequence, assays relying on effector $\mathrm{T}$-cell responses are more likely to be false-negative in young children compared with adults. In primary $\mathrm{TB}$, antigen-specific effector cells need to be newly generated, while they simply need to be reactivated and undergo clonal expansion from the memory pool in reactivation or reinfection $\mathrm{TB}$ seen in adults. The limited findings of lower sensitivity of T-SPOT.TB in EPTB versus PTB support the hypothesis that initial immune responses might be compartmentalised at the site of disease [21] and sufficient numbers of antigen-specific effector T-cells still have to evolve in the peripheral circulation to be measurable in T-cell assays. The current authors have some evidence that an initially negative T-SPOT.TB can become positive during treatment, and longitudinal follow-up of paediatric patients is a part of ongoing studies (data not shown).

However, the relatively poor performance of IGRAs in accurately predicting active, culture-confirmed TB at the time of clinical presentation remains a particular concern. In the present study, the QFG-IT performed significantly better in culture-proven TB than the T-SPOT.TB (QFG-IT 80\% versus TSPOT.TB $58 \%$; $=0.012$ ). Sensitivity for active TB in children was previously reported to be as high as $83 \%$ using the ELISPOT method and both recombinant protein as well as a range of overlapping peptides of ESAT-6 and CFP-10 [10]. A subsequent study by NicOL et al. [11] found the sensitivity to be $70 \%$ for clinical TB. Neither study used the commercially available TSPOT.TB assay and both were carried out in the hospital setting in South Africa, where children are likely to present at a later stage of their disease compared with the European setting. The length of time to disease progression might influence the magnitude of measurable immune responses. Both studies compared ELISPOT with TST; QFG-IT was not included.

The presence of a third antigen (TB7.7) in addition to ESAT-6 and CFP-10 in the QFG-IT whole blood assay might have enhanced the sensitivity of this assay in comparison to TSPOT.TB. Furthermore, additional factors, such as modulating cytokines, could play a role in whole blood assays. Such factors are no longer present when removing the plasma component for preparation of PBMC. Other groups have reported significant differences in cytokine production between whole blood assays and PBMC preparations [22, 23].

The TST was found to have better sensitivity than IGRAs for predicting definite TB and the data obtained by the current authors provide no reason to abandon the TST as a diagnostic tool for active PTB in nonimmunocompromised children. Sensitivity is clearly increased when lower cut-off values are used for the TST, but this is at the expense of specificity, which is why it was decided to apply the most restrictive cut-off point of $15 \mathrm{~mm}$ to compare sensitivities and concordance.

It is well known that a TST can be negative in EPTB, and both TST and T-SPOT.TB lost sensitivity in the context of EPTB, unlike the QFG-IT assay, which was consistent in either manifestation of TB disease.

According to the present results a negative IGRA does not exclude active TB, and use of either IGRA cannot be recommended as a single tool to confirm or rule out active TB in children. However, as an additional test it can contribute value to the overall diagnostic process and the combination of TST and IGRA reached $100 \%$ in the definite case group. Furthermore, the current authors suggest that an initially negative IGRA should be repeated in cases of presumed active $\mathrm{TB}$ in which the clinicians felt certain enough to start the child on TB treatment. Several recent studies in adults have examined the predictive value of IGRAs in combination with the TST to exclude TB rather than to confirm it [24-26]. This analysis is difficult to conduct in paediatric populations, since there is significant overlap of symptoms with other childhood illnesses and the investigations of children do not routinely include bronchoscopy. Even in the best circumstances, bacteriological confirmation is the exception rather than the rule, and it is therefore extremely challenging to exclude TB, especially in a population with several risk factors, such as that described in the present report. Therefore, the current authors do not believe that it is possible to estimate the value of IGRAs and TST in excluding active TB in such a population.

The present data show that $79 \%$ of all children who were not commenced on TB therapy (possible TB cohort) by their paediatrician had a negative IGRA or TST, somewhat justifying the therapeutic decision. Conversely, $7 \%$ and $11 \%$ of children tested with QFG-IT and T-SPOT.TB, respectively, had a positive IGRA and were not treated. Given the high suspicion of TB in this group, the positive test may have indicated LTBI. Longitudinal follow-up studies of large cohorts are required to determine the positive predictive value of IGRAs in this circumstance. A recent publication by BAKIR et al. [27] showed that children with a positive IGRA had a 3- to 4-fold increased risk of progression to active $\mathrm{TB}$, but the study could not detect a significant difference in the positive predictive value between TST and IGRAs, due to the limited numbers of children developing active TB.

In the present cohort, as well as in other published studies $[5,28,29]$, significantly fewer children were found positive on either IGRA compared with the TST. Applying the results of IGRAs rather than the TST to guide prophylactic treatment, as recommended by the NICE guidelines, would have led to a reduction of chemoprophylactic therapy by $\sim 30 \%$ in the present study cohort. However, there are no data assessing the incidence of active TB in children following a contact with smear-positive TB, who have a negative IGRA and do not receive chemoprophylaxis. To establish the negative predictive value of IGRAs in LTBI in children will require national and 
international collaborative prospective studies in settings of low and high endemicity.

Interestingly, a higher proportion of positive TST was not seen in those children who had received BCG vaccination, even accounting for different cut-offs according to national guidelines. Although somewhat contradictory to reports from adult studies [6, 30], the same has been reported from other paediatric cohorts in TB endemic countries [31, 32], in which it was concluded that BCG vaccination was not an important cause of false-positive TST, except in communities with low TB prevalence. The findings of the present study are consistent with these observations.

In LTBI, the agreement between both IGRAs was very good ( $\kappa$ 0.82) and one IGRA cannot be recommended over the other in this context. The findings mirror the recently published work by CONNELL et al. [3], which showed a concordance of 93\% between QFG-IT and T-SPOT.TB in LTBI in children and a $\kappa$ of 0.83 , compared with a concordance of $92 \%$ and a $\kappa$ of 0.82 in the present study.

According to the present data, absolute values of either IGRA do not distinguish active from latent $\mathrm{TB}$, in line with the published literature [11]. Although significant differences in induration between children with active and latent TB using TST were described, the considerable overlap and the lack of a defined cut-off between the groups makes it impossible for clinicians to distinguish the conditions via TST, which has been a long standing observation.

Although a sizeable population of children was enrolled, it could not be determined if IGRAs perform reliably in all age groups. The performance of IGRAs in very young children (aged $<2$ yrs) remains a concern and multicentre data sets are required to answer this question conclusively. The present study did not detect a significant difference in the age of children who failed or had an indeterminate IGRA result with either method; the percentage of indeterminate or failed IGRAs in the study is comparable to other published work [28, 33, 34].

In conclusion, a negative IGRA should not dissuade paediatricians from diagnosing and treating active TB. In the context of screening for LTBI, the use of IGRAs in place of TST would significantly reduce the number of children receiving chemoprophylaxis with good concordance between both assays.

However, to date there have been no prospective studies determining rates of active tuberculosis in children presenting with a negative interferon- $\gamma$ release assay at the time of contact screening, who as a consequence do not receive chemoprophylaxis. Many paediatricians will be reluctant to leave these children untreated, especially children aged $<2$ yrs.

\section{ACKNOWLEDGEMENTS}

Affiliations are as follows. B. Kampmann: Academic Dept of Paediatric Infectious Diseases, Wellcome Centre for Clinical Tropical Medicine and Centre for Respiratory Infection, Imperial College London, London, UK; E. Whittaker and N. Martinez-Alier: Academic Dept of Paediatric Infectious Diseases, Imperial College London, London, UK; A. Williams and B. Williams: Dept of Paediatrics, Northwest London Hospital Trusts, London, UK; S. Walters and A-M. Hutton:
Imperial College Healthcare NHS Trust, St. Mary's Campus, London, UK; A. Gordon: Wellcome Centre for Clinical Tropical Medicine, Imperial College London, London, UK; A.M. Crook: MRC Clinical Trials Unit, Medical Research Council, London, UK; S.T. Anderson: Academic Dept of Paediatric Infectious Diseases, Imperial College London, and Brighton and Sussex Medical School, University of Sussex, Brighton, UK.

The authors would like to thank the children and parents who participated in this study and the medical and nursing staff who helped with recruitment. We are grateful to B. Wall (Consultant Microbiologist, North West London Hospitals Trust, London, UK) for use of laboratory facilities at Northwick Park Hospital, as well as to A. Nunn (MRC, London) for helpful statistical advice.

\section{REFERENCES}

1 Marais BJ, Graham SM, Cotton MF, Beyers N. Diagnostic and management challenges for childhood tuberculosis in the era of HIV. J Infect Dis 2007; 196: Suppl. 1, S76-S85.

2 Starke JR. Diagnosis of tuberculosis in children. Pediatr Infect Dis J 2000; 19: 1095-1096.

3 Connell TG, Ritz N, Paxton GA, et al. A three-way comparison of tuberculin skin testing, QuantiFERON-TB gold and T-SPOT.TB in children. PLoS One 2008; 3: e2624.

4 National Institute for Health and Clinical Excellence. Clinical Diagnosis and Management of Tuberculosis, and Measures for its Prevention and Control. London, National Institute for Health and Clinical Excellence, 2006. Available from: www.nice.org.uk/Guidance/CG33/NiceGuidance/ pdf/English.

5 Dominguez J, Ruiz-Manzano J, De Souza-Galvao M, et al. Comparison of two commercially available $\gamma$ interferon blood tests for immunodiagnosis of tuberculosis. Clin Vaccine Immunol 2008; 15: 168-171.

6 Ferrara G, Losi M, D'Amico R, et al. Use in routine clinical practice of two commercial blood tests for diagnosis of infection with Mycobacterium tuberculosis: a prospective study. Lancet 2006; 367: 1328-1334.

7 Ewer K, Deeks J, Alvarez L, et al. Comparison of T-cellbased assay with tuberculin skin test for diagnosis of Mycobacterium tuberculosis infection in a school tuberculosis outbreak. Lancet 2003; 361: 1168-1173.

8 Dogra S, Narang P, Mendiratta DK, et al. Comparison of a whole blood interferon $\gamma$ assay with tuberculin skin testing for the detection of tuberculosis infection in hospitalized children in rural India. J Infect 2007; 54: 267-276.

9 Detjen AK, Keil T, Roll S, et al. Interferon- $\gamma$ release assays improve the diagnosis of tuberculosis and nontuberculous mycobacterial disease in children in a country with a low incidence of tuberculosis. Clin Infect Dis 2007; 45: 322-328.

10 Liebeschuetz S, Bamber S, Ewer K, et al. Diagnosis of tuberculosis in South African children with a T-cell-based assay: a prospective cohort study. Lancet 2004; 364: 2196-2203.

11 Nicol MP, Pienaar D, Wood K, et al. Enzyme-linked immunospot assay responses to early secretory antigenic target 6, culture filtrate protein 10, and purified protein derivative among children with tuberculosis: implications 
for diagnosis and monitoring of therapy. Clin Infect Dis 2005; 40: 1301-1308.

12 Lalvani A. Diagnosing tuberculosis infection in the 21st century: new tools to tackle an old enemy. Chest 2007; 131: 1898-1906.

13 Menzies D, Pai M, Comstock G. Meta-analysis: new tests for the diagnosis of latent tuberculosis infection: areas of uncertainty and recommendations for research. Ann Intern Med 2007; 146: 340-354.

14 Health Protection Agency. Tuberculosis in the UK. Annual Report on Tuberculosis Surveillance and Control in the UK 2007. London, Health Protection Agency, 2007.

15 Altman DG. Some common problems in medical research. In: Altman DG, ed. Practical Statistics for Medical Research. Boca Raton, Chapman and Hall, 1994; pp. 403-409.

16 Marais BJ, Gie RP, Hesseling AC, et al. A refined symptombased approach to diagnose pulmonary tuberculosis in children. Pediatrics 2006; 118: e1350-e1359.

17 Beyers N, Gie RP, Schaaf HS, et al. A prospective evaluation of children under the age of 5 years living in the same household as adults with recently diagnosed pulmonary tuberculosis. Int J Tuberc Lung Dis 1997; 1: 38-43.

18 Upham JW, Rate A, Rowe J, et al. Dendritic cell immaturity during infancy restricts the capacity to express vaccinespecific T-cell memory. Infect Immun 2006; 74: 1106-1112.

19 Upham JW, Lee PT, Holt BJ, et al. Development of interleukin-12-producing capacity throughout childhood. Infect Immun 2002; 70: 6583-6588.

20 White GP, Watt PM, Holt BJ, et al. Differential patterns of methylation of the IFN-gamma promoter at CpG and nonCpG sites underlie differences in IFN-gamma gene expression between human neonatal and adult CD45ROT cells. I Immunol 2002; 168: 2820-2827.

21 Wilkinson KA, Wilkinson RJ, Pathan A, et al. Ex vivo characterization of early secretory antigenic target 6specific $\mathrm{T}$ cells at sites of active disease in pleural tuberculosis. Clin Infect Dis 2005; 40: 184-187.

22 Yancy H, Ayers SL, Farrell DE, et al. Differential cytokine mRNA expression in swine whole blood and peripheral blood mononuclear cell cultures. Vet Immunol Immunopathol 2001; 79: 41-52.

23 Silberer J, Ihorst G, Kopp MV. Cytokine levels in supernatants of whole blood and mononuclear cell cultures in adults and neonates reveal significant differences with respect to interleukin-13 and interferon- $\gamma$. Pediatr Allergy Immunol 2008; 19: 140-147.

24 Diel R, Loddenkemper R, Meywald-Walter $\mathrm{K}$, et al. Predictive value of a whole blood IFN- $\gamma$ assay for the development of active tuberculosis disease after recent infection with Mycobacterium tuberculosis. Am J Respir Crit Care Med 2008; 177: 1164-1170.

25 Dosanjh DP, Hinks TS, Innes JA, et al. Improved diagnostic evaluation of suspected tuberculosis. Ann Intern Med 2008; 148: 325-336.

26 Goletti D, Stefania C, Butera O, et al. Accuracy of immunodiagnostic tests for active tuberculosis using single and combined results: a multicenter TBNET-Study. PLoS One 2008; 3: e3417.

27 Bakir M, Millington KA, Soysal A, et al. Prognostic value of a T-cell-based, interferon- $\gamma$ biomarker in children with tuberculosis contact. Ann Intern Med 2008; 149: 777-786.

28 Connell TG, Curtis N, Ranganathan SC, et al. Performance of a whole blood interferon- $\gamma$ assay for detecting latent infection with Mycobacterium tuberculosis in children. Thorax 2006; 61: 616-620.

29 Taylor RE, Cant AJ, Clark JE. Potential effect of NICE tuberculosis guidelines on paediatric tuberculosis screening. Arch Dis Child 2008; 93: 200-203.

30 Nienhaus A, Schablon A, Diel R. Interferon- $\gamma$ release assay for the diagnosis of latent TB infection - analysis of discordant results, when compared to the tuberculin skin test. PLoS One 2008; 3: e2665.

31 Araujo Z, de Waard JH, de Larrea CF, et al. The effect of Bacille Calmette-Guerin vaccine on tuberculin reactivity in indigenous children from communities with high prevalence of tuberculosis. Vaccine 2008; 26: 5575-5581.

32 Hill PC, Brookes RH, Adetifa IM, et al. Comparison of enzyme-linked immunospot assay and tuberculin skin test in healthy children exposed to Mycobacterium tuberculosis. Pediatrics 2006; 117: 1542-1548.

33 Ferrara G, Losi M, Meacci M, et al. Routine hospital use of a new commercial whole blood interferon- $\gamma$ assay for the diagnosis of tuberculosis infection. Am J Respir Crit Care Med 2005; 172: 631-635.

34 Pai M, Lewinsohn DM. Interferon- $\gamma$ assays for tuberculosis: is anergy the Achilles' heel? Am J Respir Crit Care Med 2005; 172: 519-521. 